\title{
Epidemiology and community health in the medical curriculum: the Nottingham experience
}

\author{
J M ELWOOD and the academic staff of the Department of Community Health, University of Nottingham, \\ Queen's Medical Centre, Nottingham NG7 2UH, England
}

SUMMARY The epidemiology and community health teaching programme in the medical curriculum in Nottingham is described. Epidemiology is taught as a major scientific discipline in the first two years, in addition to being applied to clinical situations in the later years. All students undertake a research project in the third year, and examples of these are given.

This report describes the teaching of the Department of Community Health in the medical course at the University of Nottingham, England. The programme is unusual among those in British medical schools in that epidemiology is regarded as a basic medical science, providing a complementary methodology and approach to that of laboratory based sciences in training future physicians, in addition to being used as a clinically applied methodology. This approach and the opportunities provided in a new medical school have led to the development of a programme which is substantially different from that in most other schools.

\section{The Nottingham Medical School}

The medical school started in 1970 , being the first new British school this century, and therefore was preceded by considerable discussion of the characteristics of the curriculum. ${ }^{1}$ Nottingham is unique in Britain in that each student of medicine spends most of an academic year pursuing advanced work and a research project in one department of the student's choice, and every student is therefore awarded at the end of the third year a classified honours Bachelor of Medical Science (B Med Sci) degree. After a further two years of clinical training the student is awarded the graduating qualification in medicine of Bachelor of Medicine, Bachelor of Surgery (BM,BS). He or she is then eligible to undertake a preregistration house physician or house surgeon appointment and after successfully completing this one year experience, is eligible for full registration with the General Medical Council.

The current intake is approximately 130 students. Male and female applications are assessed without regard to sex, and usually about half of each intake is female, one of the highest proportions in the United
Kingdom. For several years the usual entry requirement was three science subjects at Advanced level of the General Certificate of Education with grade B or better; of the 1984 intake, half had three A grades.

The Department of Community Health was one of the six foundation departments of the new medical school. It is responsible for teaching community medicine, epidemiology, biostatistics, general practice, occupational medicine, preventive medicine, and health education. The current staff includes one professor and five senior lecturers, medical staff at this level having consultant status, three lecturers, 15 part time lecturers in general practice, and four external lecturers. The overall structure of the course is shown in the figure. The department makes a major contribution to the first three years and has a smaller component of the clinical teaching. The teaching of general practice will not be fully described here, only those aspects relating to community health and epidemiology being covered.

The Department of Psychiatry has a behavioural sciences division which contributes to teaching on socioeconomic and cultural differences in health knowledge and beliefs, the sick role, and responses to illness.

Basic statistics is taught as an interdisciplinary course linked to training in laboratory and clinical measurement.

COURSE CONTENT AND METHODS OF TEACHING: PRECLINICAL YEAR

During the first two years (6 terms) there are 91 hours of timetabled commitment to the Department of Community Health for each student. Only 27 of these hours are taken up by lectures, 25 being seminars in groups of 10 to 25 , and 39 hours for 


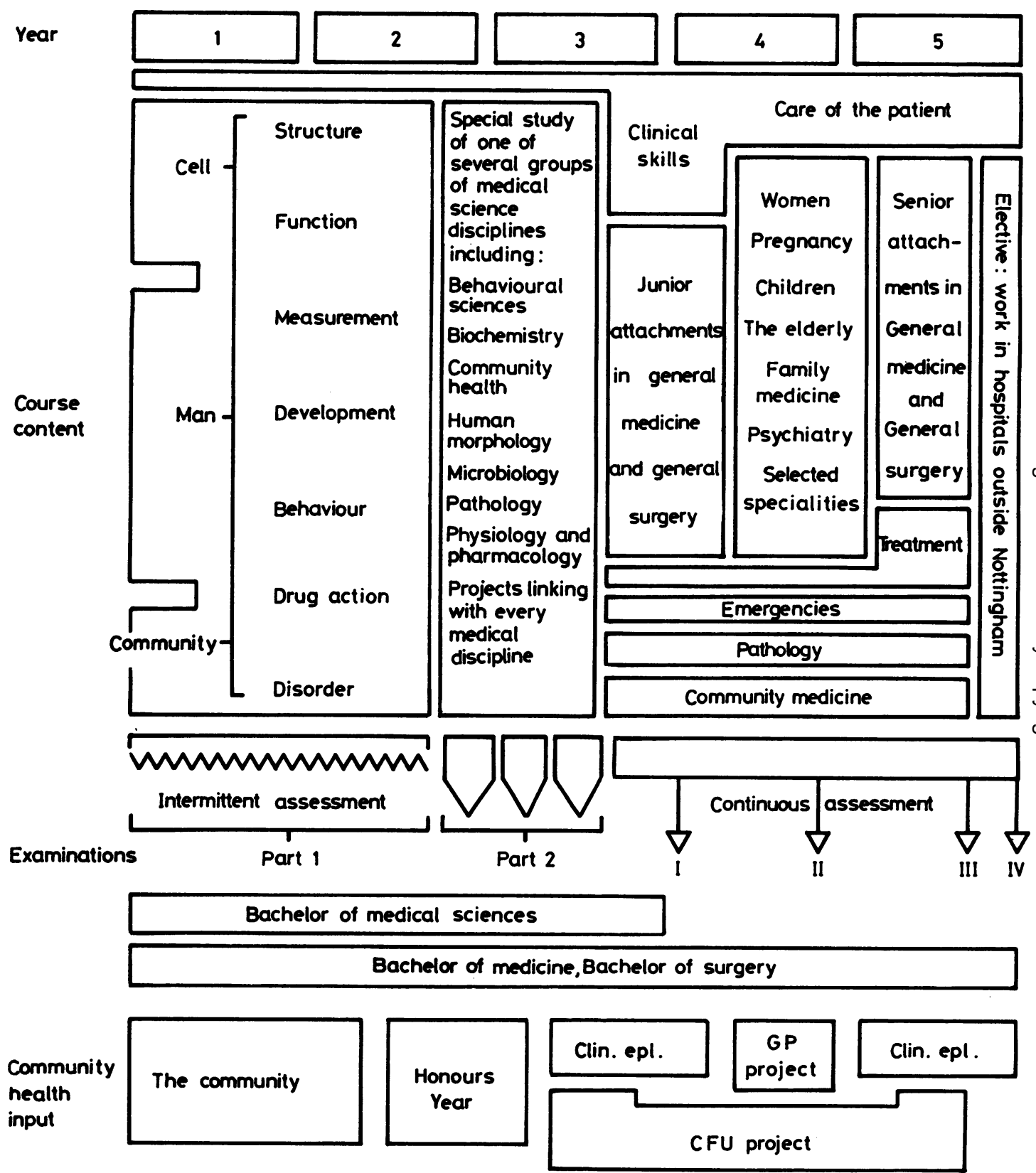

Fig 1 General structure of the Nottingham medical course, and the contributions from the Department of Community Health. 
private study in connection with projects and individual visits. The structure of the course is illustrated in table 1 . On the first two days of the course, students visit a general practice and a hospital ward. In the first term, four lectures and four seminar exercises deal primarily with descriptive epidemiology, making students familiar with terminology, routine sources of information, and the interpretation of tabular and graphical material. These sessions give the opportunity to include knowledge about the factors affecting the health of communities and the historical trends in mortality and morbidity over the last 150 years. Differences in demographic features and in causes of major morbidity and mortality between countries are also dealt with. During the first term students are taught basic statistical techniques in the context of a laboratory based course entitled 'Measurement in Medicine', and this is built on in the community health course by use of the statistical techniques necessary to compare incidence and mortality rates.

Most of the systematic teaching is done in seminar groups of 20-25 students, and carefully prepared exercises have been developed. These either use the conventional written format where students are given a handout with raw data and questions, or use specially prepared, video-taped material, in which a presentation is made of original data, quotations from papers, and suitable illustrative material to give the students a detailed but carefully rehearsed introduction to a specific topic. At the end of the first term students are introduced to the ideas of cohort and case control studies and randomised trials, as they meet these concepts in the second term project, and a lecture is given which illustrates current applications of epidemiological methods.
In the second term, five groups of approximately 25 students are set up, and each group studies one disease in detail. They do this under the guidance of a medically qualified tutor, and the first seminar involves meeting a patient who has been affected by the disease and discussing the clinical, social, and personal aspects of the disease with the patient. Then each student is given a specific epidemiological topic on which to produce a written paper. For example, for a session on breast cancer, questions on different epidemiological aspects of breast cancer are given, such as "Is there a relation between high fat diet and the occurrence of breast cancer?" and "What is the evidence that mammographic screening is valuable?" Students prepare a written answer to the question in their own time over some four weeks and also prepare a 5-10 minute oral presentation. At a further seminar students present the results of their own researches, all of which relate to the same disease, and therefore have some overlapping concerns, and the different issues raised are discussed. This exercise is a popular one with both staff and students, and the standard of the written reports is generally high. It is the first opportunity in the curriculum for the students to search out and read original material from the library, and one of the first opportunities for them to display their ability to evaluate critically and synthesise original work.

During the term also, lecturers deal with the history and current structure of the National Health Service and compare this with health services in other countries such as the USA, a western and an eastern European country, and a developing country. These presentations are made by internal or guest lecturers who have personal experience of working in the countries concerned. The other topic emphasised in

Table 1 Teaching in the first two years

\begin{tabular}{|c|c|c|c|c|c|}
\hline Year & Term & Topics & Lectures & Seminars & $\begin{array}{l}\text { Individual } \\
\text { study }\end{array}$ \\
\hline 1 & 1 & Visits to hospitals and general practice & 1 & & 6 \\
\hline 1 & 1 & $\begin{array}{l}\text { Major causes of morbidity and mortality } \\
\text { Historical changes } 1800-1984 \\
\text { Descriptive epidemiology; vital statistics, demography; rates, comparisons between populations } \\
\text { Statistical inference } \\
\text { Introduction to analytical epidemiology }\end{array}$ & 7 & 8 & 4 \\
\hline 1 & 2 & $\begin{array}{l}\text { Medical care provision in the UK and other countries } \\
\text { Socioeconomic factors in health } \\
\text { Disease epidemiology: study of one disease in depth (project) }\end{array}$ & 8 & 5 & 10 \\
\hline 1 & 3 & No teaching & & & \\
\hline 2 & 4 & Epidemiological methods: analytical and experimental studies; assessment of causal relations & 4 & 6 & \\
\hline 2 & 5 & Assessment of one issue in health care provision or in prevention (project) & & 6 & 11 \\
\hline \multirow[t]{2}{*}{2} & 6 & $\begin{array}{l}\text { Delivery of medical care: general practice, hospitals, occupational health, community care, } \\
\text { preventive services } \\
\text { Effect of individual behaviour on health; health education, behavioural change, evaluation of } \\
\text { preventive programmes. }\end{array}$ & 7 & & 8 \\
\hline & & Totals & 27 & 25 & 39 \\
\hline
\end{tabular}


this term is socioeconornic influences on disease and on health care provision and utilisation; this is coordinated with teaching on socioeconomic and cultural differences in health knowledge and beliefs, the sick role, and responses to illness, given by the behavioural sciences department.

There is no community health teaching in the third term, and in the first term of the second year students deal with epidemiological methods in considerably more detail, through a series of four lectures on analytical epidemiology methods and three seminar exercises, two using video tape presentations and one using written material. By the end of the fourth term students are expected to show familiarity with the major study approaches of analytical epidemiology, which we take to include randomised trials. They are also expected to be able to evaluate evidence for a relation between an exposure and an outcome variable in terms of criteria for causality, and by a systematic effort to evaluate possible non-causal associations due to observation bias, confounding, or chance variation. The details of methods to control confounding, such as matching, stratified analysis or multivariate analysis, are regarded as beyond the scope of students at this stage.

In the fifth term, presentations by outside and internal speakers deal with the provision of care in different settings such as general practice, occupational health, hospital, and community based care. Also a programme is given dealing with the factors influencing health related behaviour, such as smoking or seat belt use, and in particular the role of the physician in modifying such behaviour. The ways in which primary prevention can be achieved are discussed, including legislative and community based actions, mass media health education, and individual approaches.

In the sixth term students undertake, in groups of about 10 , a project which usually has a health care delivery focus, and the primary emphasis in this project is that of using the opportunity to make visits to community based services and discuss the provision of care services for special groups of people, such as those with specific handicaps or for specific topics. There is no specific epidemiological component in this project, although clearly the student's epidemiological training is brought into play.

\section{THE HONOURS YEAR}

From June of the second year to April of the third year, each student works in a department of his or her choice pursuing a course of advanced study, including a research project which is presented as a dissertation. The Department of Community Health currently accepts approximately 12 students per year, and in recent years up to twice this number have given the department as their first choice. The assignment to departments is made by the students through their own representatives. The programme in the department consists of a six week course in research methods, $4 \frac{1}{2}$ months work on the student's dissertation, and a third module addressing issues in community health not covered elsewhere in the course (table 2).

The research methods course deals with epidemiological and statistical methods and methods of data collection, and includes seminars in which 8 members of staff and guest speakers discuss their current research activities. Eight seminars of $1 \frac{1}{2}$ hours each deal with the design, analysis, and interpretation of analytical epidemiological studies, including methods of controlling confounding, matched and unmatched analysis, lifetable methods, and an introduction to multivariate methods.

A similar number of seminars on statistics deal with non-parametric and parametric methods,

Table 2 Teaching in the Honours year

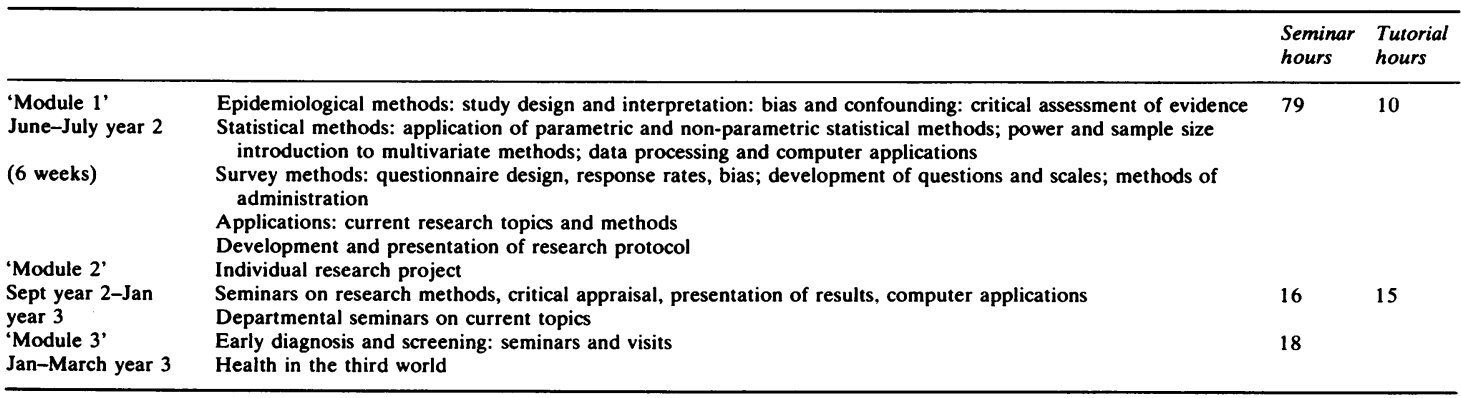


regression and correlation methods, statistical inference, and power and sample size. A series of computer based exercises on either hypothetical or real data, giving the student the experience of accessing a data file, generating tabulations on an interactive computer system, and applying appropriate statistical tests, brings together the epidemiological and statistical parts of the programme and helps the student to gain familiarity with computer applications.

A third major series of seminars deals with information gathering techniques, including questionnaire design and survey methods, and discusses the application of methods such as participant observation and the use of audio and visual recording. During this six week module, each student also develops the protocol for his or her project, under the guidance of an individual tutor, and presents a developed protocol for the project to fellow students and staff members at combined sessions. These protocol discussion sessions, at which each student usually has half an hour of discussion, are given great importance, and acceptance of the protocol constitutes the department's approval of the scientific, ethical, and practical aspects of the project. Each protocol includes an assessment of ethical issues and those concerned with confidentiality and professional etiquette, as well as a budget statement. For most projects formal external ethical review is not required, but exceptions include projects which involve access to records in general practice or in certain areas of hospital care, or involve a measure of experimentation.

In contrast to the laboratory based departments in the medical school, which usually provide students with a finite list of projects from which they choose, students entering the Department of Community Health are encouraged to develop their own ideas as far as possible. The department also provides a quite extensive list of potential research topics related to current activities. Usually some students pursue topics outside Nottingham or overseas, those going overseas having taken the initiative to find financial and professional support well in advance. Table 3 shows the topics chosen by students who graduated in 1984.

The third portion of the Honours course covers the time interval between the submission of the dissertation and its assessment at the end of the academic year, and currently this programme consists of seminars and visits on two topics, one being health care provision in the third world and the other issues of prompt diagnosis and screening in British practice.

The third year culminates in the award of a Bachelor of Medical Sciences degree, which is awarded where appropriate with First, Upper Second, Lower Second or Third Class Honours. Some $25 \%$ of the marks contributing to this degree are based on the student's performance in the first two years in all subjects in the curriculum, and the remainder on activities in the Honours year, of which the dissertation forms a large part. Examinations are conducted by internal and external examiners. The standard of dissertations, in the opinion of both departmental staff and our external advisers, is high (the best being scientifically equivalent to those seen in Master's programmes or submissions for the MFCM qualification). The dissertations are obviously constrained by the short time available, and therefore few equate in terms of breadth or quantity of new information with those for

Table 3 Topics of students' dissertations in the third year, 1983-4

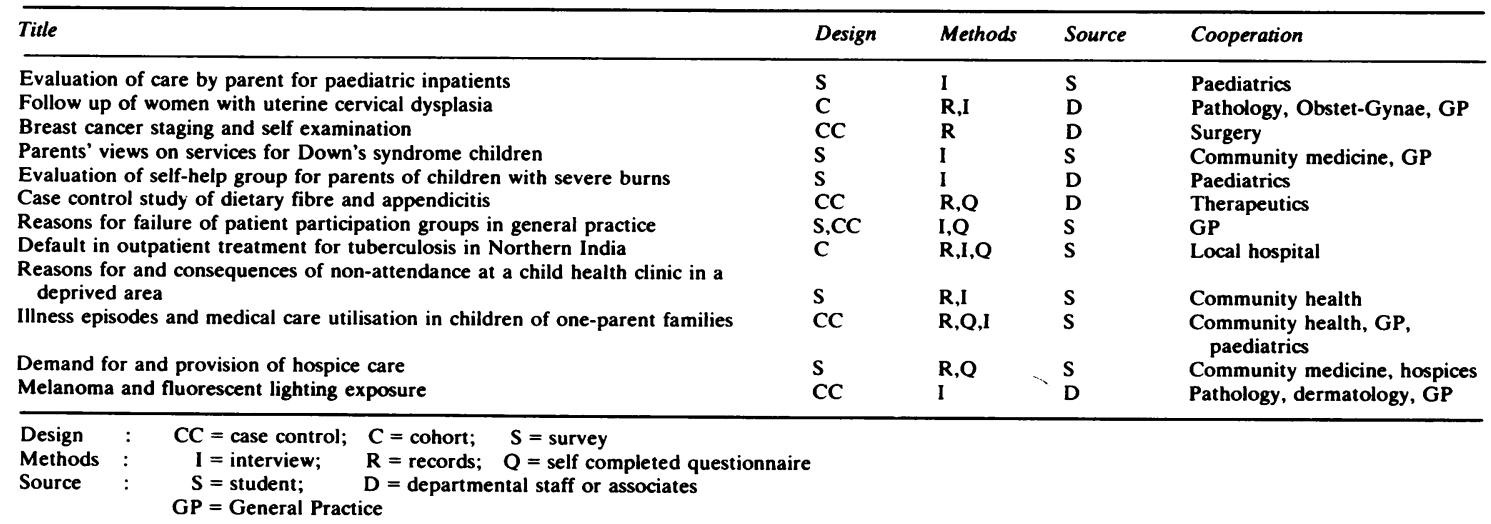


higher degrees. About half of the dissertation projects in recent years have resulted in publications in refereed journals or presentations at major meetings, either as independent pieces of work or in combination with other work.

\section{THE CLINICAL PROGRAMME}

Students enter the clinical programme in May of the third year, graduating in June of the fifth year. Because the Nottingham programme includes the Honours research year within the overall constraints of a five year programme the clinical teaching is compressed, and students have only a short vacation period and a one month elective period during their clinical training.

Epidemiological input to the clinical programme is in two parts: a course of seminars in clinical epidemiology and a project (table 4). The seminar course is given partly during the first six months of the junior clinical year, and the second part in the senior year a few months before graduation, and emphasises the critical appraisal of written and oral information and of the physician's own clinical experience. The topics include the use of diagnostic tests, error and bias in clinical measurement, randomised and non-randomised comparisons of treatment modalities, screening, and evaluation of the current medical literature. The seminars in the final year tend to deal with topics of current controversy; in 1983 these included vitamin supplementation to prevent neural tube defects, oral contraception in relation to breast and cervical cancer, and options in drug treatment to prevent reinfarction.

The community follow-up project is designed to emphasise the relation between hospital and community care and to help the student to see a patient's disease problem in the family and social context. The project has been described previously ${ }^{2}$. Each student studies one patient with chronic disease who is seen in hospital during the student's first clinical attachment, and who is subsequently discharged. The student follows the patient's progress over $1 \frac{1}{2}$ to 2 years by visiting the family at home and visiting the general practitioner and others concerned with the patient's care. The student prepares a report, documenting the hospital and community aspects of the patient's care and including a review of the epidemiology and natural history of the patient's condition. These reports frequently document failures of the health care system, particularly communication difficulties between hospital and community care, and some have influenced changes in the way care is provided in hospital units.

The department also contributes regular teaching to the obstetrics-gynaecology and child health programmes. During the student's one month attachment to general practice an evaluative or audit project is carried out. These vary considerably in scope but recently have included studies of vaccinations, cervical cytology, and digoxin treatment. They require the student to apply to a practical medical care situation many of the epidemiological concepts they have learned in earlier years.

\section{ST A F F I N G}

The staffing requirements of this input into all five years of the medical course, for one calendar year, are given in table 5. Approximately 600 person-hours of teaching time per year are required from senior staff, that is, full time or major part time regular members of the academic staff of the department at lecturer level or above. Currently there are nine such staff, three having joint appointments, giving 7.5 full time equivalent staff with on average 82 hours per year timetabled teaching time. Much time is also needed for the setting and marking of assessments, a considerable amount of informally arranged teaching, and regular input to the interdepartmental introductory course in the first year and to the programmes of other departments.

In addition, about 280 person-hours of teaching are given by part time lecturers in general practice for the community follow-up programme, by tutors in first and second years, and by visitors and hosts of student visits.

Table 4 Teaching in the clinical years

\begin{tabular}{ll}
\hline & \\
\hline 'Junior attachment' & Community follow up (CFU): hospital, community and general practice care; epidemiology \\
April 3 year-Oct 4 year & Clinical epidemiology: diagnostic tests and decision making; screening; clinical trials \\
'Middle year' & Audit project in general practice attachment \\
$\begin{array}{l}\text { Oct } 4 \text { year-5 year } \\
\text { 'Senior year' } \\
\text { Oct } 5 \text { year-June } 5 \text { year }\end{array}$ & CFU further visits and conclusion \\
\hline
\end{tabular}


Table 5 Staffing requirements for one calendar year

\begin{tabular}{|c|c|c|c|c|c|c|}
\hline & \multicolumn{3}{|c|}{ Senior staff } & \multicolumn{3}{|c|}{ Associate staff, part time staff, and visitors } \\
\hline & Hours & Persons & $\begin{array}{l}\text { Person } \\
\text { hours }\end{array}$ & Hours & Persons & $\begin{array}{l}\text { Person } \\
\text { hours }\end{array}$ \\
\hline \multicolumn{7}{|l|}{ First/Second Year } \\
\hline $\begin{array}{l}\text { Lectures } \\
\text { Seminars Terms } 1,4\end{array}$ & 25 & 1 & 25 & $7 \frac{1}{2}$ & 2 & 15 \\
\hline Seminars Terms 1,4 & 14 & 1 & 14 & 14 & 5 & 70 \\
\hline Seminars Term 2 & 4 & 5 & 20 & & & \\
\hline Seminars Term 5 & 6 & 2 & 12 & 6 & 6 & 36 \\
\hline \multicolumn{7}{|l|}{ Third (Hon) Year } \\
\hline \multicolumn{7}{|l|}{ Module 1} \\
\hline Seminars & 57 & 1 & 57 & & & \\
\hline Presentations & $22 \frac{1}{2}$ & 6 & 135 & & & \\
\hline Tutorials & 10 & 10 & 100 & & & \\
\hline \multicolumn{7}{|l|}{ Module 2} \\
\hline Seminars & 16 & 1 & 16 & & & \\
\hline Tutorials & 15 & 10 & 150 & & & \\
\hline \multicolumn{7}{|l|}{ Module 3} \\
\hline Seminars & 9 & 1 & 9 & 24 & 1 & 24 \\
\hline Visits & & & & 12 & 1 & 12 \\
\hline Presentations & 9 & 2 & 18 & & & \\
\hline \multicolumn{7}{|l|}{ 'Junior' Clinical } \\
\hline Epidemiology seminar & 5 & 4 & 20 & & & \\
\hline Community follow-up seminars & 5 & 3 & 15 & 5 & 15 & 75 \\
\hline \multicolumn{7}{|l|}{ 'Senior' Clinical } \\
\hline Epidemiology seminars & 4 & 4 & 16 & & & \\
\hline Senior community follow-up & 3 & 3 & 9 & 3 & 15 & 45 \\
\hline Totals & & & 616 & & & 277 \\
\hline
\end{tabular}

Note: Excludes audit project in general practice

\section{Discussion}

The Nottingham course is unusual in the proportion of curricular time devoted to topics in epidemiology and community health, and in the intensity with which these topics can be dealt, particularly in the preclinical years. The programme makes extensive demands of staffing resources, as indicated in table 5 . First and second year students work from October to May, third year students from June to March, with the exception of the month of August, and clinical students are present all year round so that the department is involved in teaching continuously. The sections of the programmes which are dealt with in seminars and small group sessions are very demanding in terms of staff time. As shown in the table, we are heavily dependent on the availability of tutors in various stages of the programme. For the first and second year teaching, assistance is given by registrars and senior registrars in community medicine, postgraduate students in the department, some consultant level staff in community medicine and related subjects, and National Health Service non-medical staff with statistical or epidemiological expertise. In the clinical years, the community follow-up project and associated seminars are given by our staff of part-time lecturers in general practice.
The clinical epidemiology seminars raise difficulties in the timing and site of the teaching sessions, and it has been difficult to bring in help from outside. As a result these sessions are currently being run with groups of 20-30 students, which is too large a number for a true seminar approach to be adopted. Assistance from outside the department is provided primarily through good-will and mutual interest, although a modest financial remuneration is given and is essential for the regular continuation of the programmes.

No formal evaluation of the components or of the total programme has been made. There is a system of feedback from the students both through committees and more informally, and surveys of students' and teachers' reaction to segments of the programme have been used regularly in the general practice attachment and intermittently in various other aspects of the programme. In general we feel that the preclinical programme in the first two years is successful in creating enthusiasm and interest for the subject. It has the advantages of being clearly clinically related as much as, or more so than, the other subjects such as physiology, anatomy, and biochemistry which the students are studying at that time. Our subjective impression over the last few years, during which we have increased the depth of 
study required into epidemiological methods and made the course considerably more intellectually demanding, has been that these changes have made the course more attractive to our students.

The honours year seems highly successful, as judged by the reactions of the students who pursue the honours programme in the department and the fact that in recent years we have had a substantial oversubscription in terms of the numbers of students who wish to study in the department. The honours year in this department is considered by some students to be more demanding and challenging than the honours programme in some laboratory based departments, where the student is given less freedom in terms of choice or conduct of a project.

The general practice one month attachment is also regarded as one of the most popular aspects of the Nottingham course. The proportion of Nottingham students electing general practice as a first option after graduation is among the highest in Britain, and this is probably a selection effect in that the commitment to general practice and the community approach to disease is emphasised considerably in the prospectus which is available to students considering entry, and a high proportion of students at pre-entry interview indicate an interest in general practice. The degree of interest does not seem to be lost during the training period.

The area of the programme we have had most difficulty with has been the other clinical sessions: the clinical epidemiology seminars and the community follow-up project. In the former case this is largely a question of timetabling and conflict of interest, as the clinical epidemiology seminars, in common with seminars on several other subjects, cause difficulty by being islands of timetabled teaching during ward attachments. This inevitably leads to conflicts with the student's clinical commitments, particularly operating theatre and take-in duties. We feel that these sessions would be more successful if they could be run with smaller groups made up from students attached to only one or two clinical firms, rather than the large groups which at present demand that we bring together in one place and at one time students from 8-10 different clinical firms. However, this has been impossible because of staff availability.

The community follow-up project is regarded as a very valuable and worthwhile experience by most students once it is completed, but there is no doubt that it is not particularly popular because it involves a considerable effort by the student, requires the production of a written report by a deadline date, and again gives the student a potential conflict of interest situation in terms of organising his or her own time.

It would be most interesting to test whether the different overall emphasis of the Nottingham programme produces a difference in the knowledge attitudes or orientation of graduates. Little information on this is available. Career choice information in regard to epidemiology is particularly unhelpful. The most obvious related career choice is that of community medicine. Discussion of career choice with our students and recent graduates tends to show that, rightly or wrongly, their image of community medicine is most often one of administrative medicine, taking them away both from clinical care and from the clinically related type of epidemiology which we emphasise in our programe. We have a noticeable number of senior students and graduates who express an interest in the epidemiological approach as applied to clinical medicine and seek advice on how to develop a career in which they can continue this interest. It is unfortunate that in the current National Health Service there is no career pattern for that interest. The most viable options available to students are either to pursue epidemiology within the framework of community medicine, usually sacrificing thece clinical link, or to make a career in a clinical specialty, pursuing the epidemiological interest where there are ${ }^{+} \omega$ opportunities for research, which usually arise late in $\underset{\overrightarrow{0}}{\vec{\infty}} \overrightarrow{\mathrm{D}}$ their professional training, or eventually students $\frac{\mathrm{O}}{\mathrm{O}} \mathrm{G}$ may gain a teaching hospital type appointment in that $\stackrel{\frac{9}{P}}{\mathrm{~S}}$ clinical specialty. We do not regard the production of $\stackrel{\mathbb{Q}}{\Omega}$ professional epidemiologists or even community $\mathbb{D}$ physicians as a particular purpose of the $8 \stackrel{\mathbb{D}}{\mathbb{D}}$ department's teaching programme. The objective as뭉 we see it is to equip all our graduates with the skills of epidemiological reasoning and critical evaluation of patient based material. We would be most interested in any opportunity to compare the attitudes and orientation of the knowledge base of our graduates and those of other British universities which have large programmes of epidemiology and community medicine with graduates of more traditionally orientated programmes. Such a study would have to assess the likely differences of orientation of incoming students and would be best performed by a neutral and disinterested investigator.

This teaching programme has been developed by the current departmental staff, especially Drs MW Beaver, RFA Logan, RJ Madeley, JCG Pearson, and Ms Pamela Gillies. In addition, the programme owes 
much to former staff members, especially Emeritus Professor EM Backett, Dr (now Professor) AJ Hedley and Dr J McEwen. We would like also to thank all other staff members and those who assist in the course, and our secretarial and technical staff.

\section{References}

${ }^{1}$ Report of the Medical School Advisory Committee, University of Nottingham. Published, University of Nottingham. (1985).

${ }^{2}$ Miller DS, Backettt EM, Vaughan JP. After the hospital episode. Lancet 1975; ii: 545. 